\title{
Reconstrucción vulvoperineal con colgajos locales
}

\author{
Vulvoperineal reconstruction with local flaps
}

\author{
Maximiliano Oscar Abalos ${ }^{1}$; Nathaniel Dionisio Arellano²; Justo La Torre Vizcarra ${ }^{3}$
}

\section{RESUMEN}

Luego del tratamiento quirúrgico de la patología maligna y premaligna de la región vulvoperineal nos enfrentamos al desafío de la reconstrucción del defecto de cobertura, el cual en la mayoría de los casos no puede resolverse con un cierre primario. Se presentan 2 casos de reconstrucción con colgajos locales con el objetivo de reparar el defecto, ofrecer un tejido vascularizado, el cual aporta mayor oxigenación, migración celular y un buen resultado estético - funcional. La reparación se realizó con colgajos de trasposición dependientes de la arteria pudenda y colgajos de avance y rotación del lado contralateral. Hemos concluido que la confección de colgajos locales en la región vulvar para la reconstrucción de defectos es una opción a tener en cuenta debido al aporte vascular, la presencia de perforantes dependientes de la arteria pudenda, la conservación nerviosa, el bajo índice de morbilidad y el aceptable resultado estético - funcional.

Palabras claves: vulvoperineal, colgajos locales, maligno, defecto de cobertura.

\begin{abstract}
After the surgical treatment of the malignant and premalignant pathology of the vulvoperineal region we have the challenge of reconstruction the coverage defect, which in most cases cannot be resolved with a primary closure. We describe 2 cases of reconstruction with local flaps, the aim is to repairing the defect, offering a vascularized tissue, which provides more oxygenation, cell migration and a good aesthetic-functional result. The repair was performed with transposition flaps dependent on the pudendal artery and advance and rotation flaps of the contralateral side. We concluded that the repairwith local flaps in the vulvar region for the reconstruction of defects is an option to take into account due to the vascular contribution, the presence of perforators dependent on the pudendal artery, nerve conservation, low morbidity index and acceptable aesthetic result - functional.

Key words: vulvoperineal, local flaps, malignant, coverage defect.
\end{abstract}

REVISTA ARGENTINA DE CIRUGÍA PLÁSTICA 2020;26(1):45-48. HTTPS://DOI.ORG/10.32825/RACP/202001/0045-0048

\section{INTRODUCCIÓN}

El defecto de cobertura que provoca el tratamiento quirúrgico de la patología premaligna o maligna de la vulva resulta un desafío al momento de su reconstrucción, el cual en la mayoría de los casos involucra zonas contiguas que no pueden resolverse con un cierre primario.

Cabe agregar el efecto psicológico que conlleva un mal resultado funcional y estético de la región vulvoperineal.

Dentro del arsenal para la reconstrucción disponemos de colgajos, ya sea locales o libres, injertos autólogos o el cierre por segunda del defecto.

El objetivo este irabajo es proponer a los cirujanos plásticos la opción de realizar la reconstrucción vulvoperineal con colgajos locales ya que son versátiles, presentan una amplia red vascular y brindan un buen resultado estético funcional.

\section{MATERIALES Y MÉTODO}

Estudio retrospectivo donde describimos 2 pacientes con patología maligna vulvoperineal que han sido

1. Cirujano plástico y reconstructivo. Director médico de Exea Salud

2. Médico de planta. Servicio de Cirugía Plástica, Hospital Tornú

3. Jefe de Departamento de Cirugía, Hospital Tornú, CABA. Miembro Titular SACPER

$\square$ Correspondencia: Maximiliano Ábalos. Tel: 1164348302. maximilianoabalos@gmail.com

Los autores declaran no tener conflictos de intereses

Recibido: 14/09/2019 / Aceptado: 23/11/2019 evaluadas e intervenidas por los Servicios de Cirugía Plástica y de Oncoginecología del Hospital General de Agudos Enrique Tornú. Los procedimientos se realizaron en el periodo 2014-2015.

\section{RESULTADOS}

\section{CASO 1}

Paciente de 77 años que es derivada del Servicio de Ginecología de nuestro nosocomio con diagnóstico de enfermedad de Paget extramamaria para tratamiento en forma conjunta.

Presenta lesión plana, eritematosa y de bordes irregulares en región vulvar bilateral, que involucra el margen posterior del introito vaginal, labios mayores y menores, que se extiende a periné derecho, de unos $6 \times 4 \mathrm{~cm}$ de diámetro. La biopsia incisional informa enfermedad de Paget.

Antecedentes de hipertensión arterial y colecistectomía a los 50 años. Riesgo quirúrgico habitual.

La estrategia para la reconstrucción es dividir el defecto primario en dos, a partir de la línea media, quedando de esta manera un defecto mayor del lado derecho para el cual se planifica un colgajo de transposición desde el muslo ipsilateral y un defecto izquierdo, menor al anterior, al cual se le confeccionará uno de avance glúteo.

Luego de la marcación de los colgajos (Figura 1), el equipo de cirugía ginecológica le realizó una hemivulvectomía simple derecha con extensión izquierda.

Luego de la resección oncológica, con márgenes de piel sana de $2 \mathrm{~cm}$, y la paciente en posición de litotomía (Figuras 2 y 3), se confecciona colgajo de transposición der- 


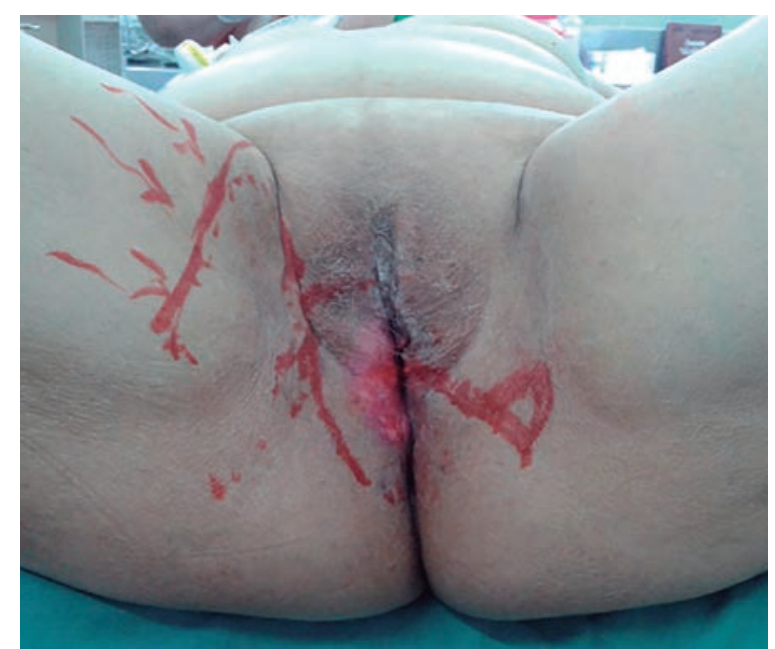

Figura 1. Marcación prequirúrgica.

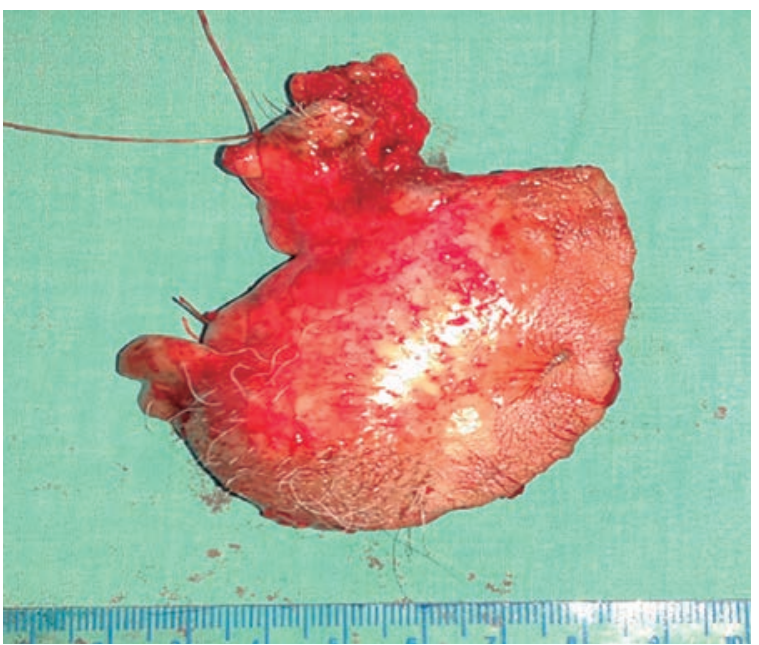

Figura 3.

mograso desde la cara interna de muslo derecho, fijando el mismo al borde libre de la mucosa vaginal (desde hora 11 a 7) sin tensión, para la mitad derecha del defecto.

Se continúa con la reparación del lado izquierdo con la confección de un colgajo dermograso de avance desde el glúteo ipsilateral hacia la mucosa vaginal (hora 7 a 1). Cierre del rafe medio. Piel con Nylon 4-0. Se coloca drenaje aspirativo. (Figura 4).

La paciente evoluciona en forma favorable, sin complicaciones, se extrae el drenaje a las 24 horas y se indica la externación hospitalaria al 4to día posoperatorio. Se realiza un seguimiento ambulatorio durante dos meses.

Se obtuvo un resultado estético y funcional satisfactorio, valorado tanto por la paciente como por el equipo quirúrgico (Figura 5).

\section{CASO 2}

Paciente de 47 años con diagnóstico de carcinoma escamoso bien diferenciado, úlcero-infiltrante, asociado a neoplasia intraepitelial vulvar diferenciada. Es evaluada interdisciplinariamente con el Servicio de Oncoginecología del Hospital Tornú. Presenta una lesión leucoplásica, indurada, de bordes poco definidos

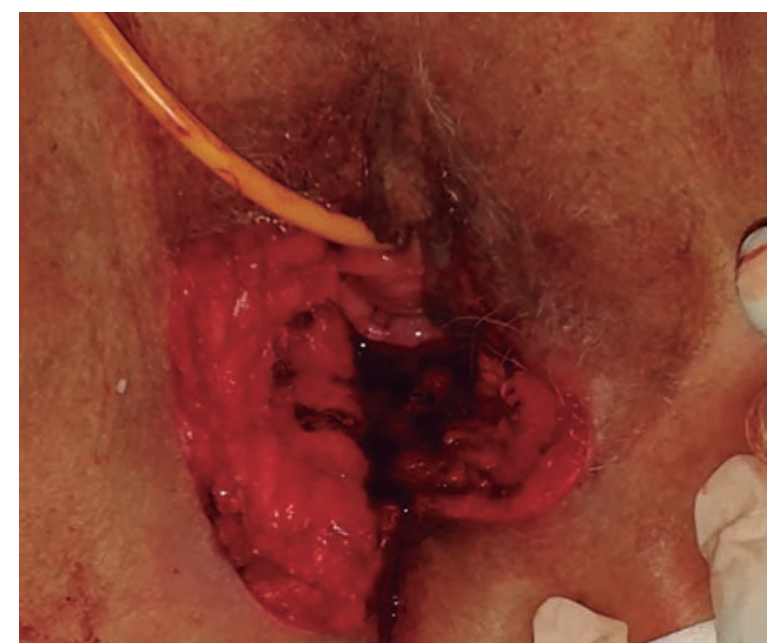

Figura 2.

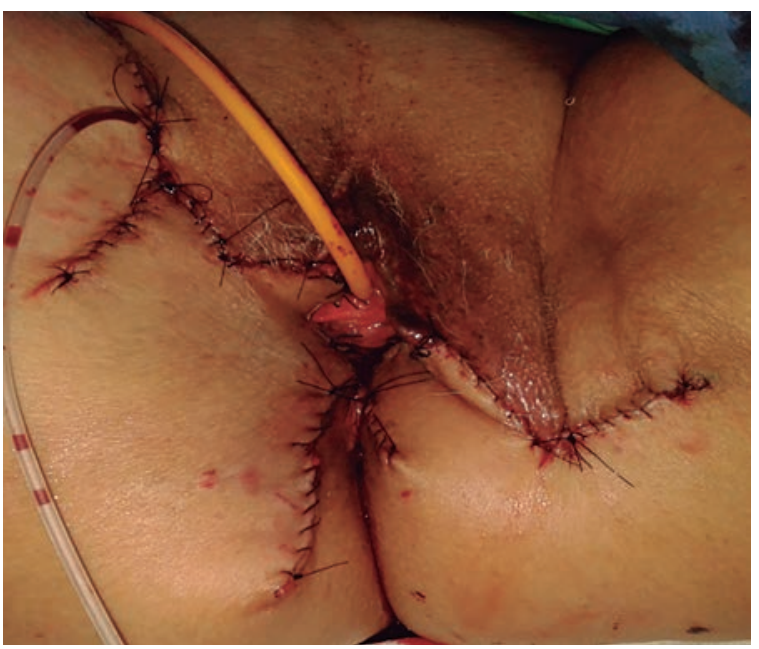

Figura 4.

en la hemivulva derecha con compromiso del introito de $4 \times 6 \mathrm{~cm}$. Compromete labio menor izquierdo y parte del labio mayor a nivel medio.

Antecedentes de anexohisterectomía por cáncer de cuello uterino en el 2002.

La estrategia para la reconstrucción en este fue diseñar un colgajo de transposición dependiente de la arteria pudenda interna del lado derecho y un colgajo dermograso en flor de loto del lado izquierdo.

Con la paciente en posición de litotomía, el equipo de cirugía ginecológica realizo una hemivulvectomía simple derecha con extensión izquierda, con márgenes libres de lesión de $2 \mathrm{~cm}$ para estudio anatomopatológico por congelación (Figura 6).

Se realizó la marcación de colgajo de trasposición dermograso dependiente de la arteria pudenda interna del lado derecho. Se disecó el colgajo hasta el plano aponeurótico (Figura 7). Una vez elevado y conservando el pedículo previamente mencionado, se fijó a la mucosa vaginal en hora 12 con sutura reabsorbible de Vicryl (Figura 8). Se realizó el cierre en dos planos a nivel lateral y en la línea media se suturó a mucosa vaginal y en hora 6 al colgajo contralateral. Se colocó drenaje aspirativo (Figura 9). 


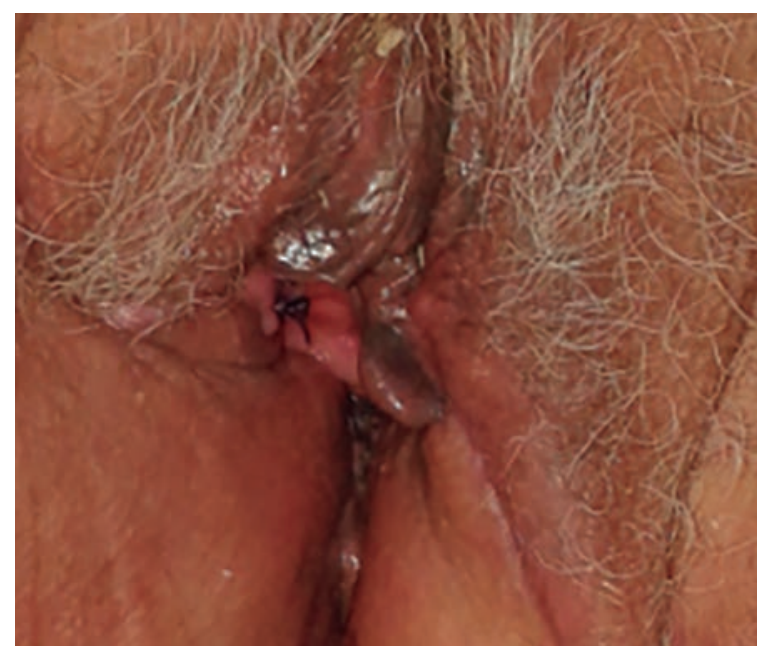

Figura 5.

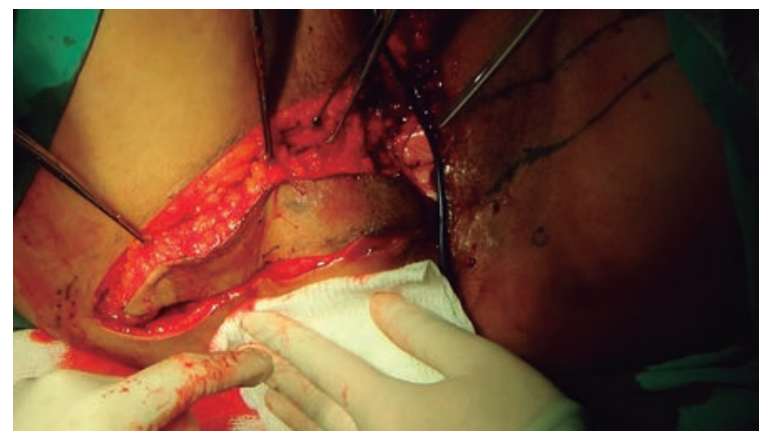

Figura 7.

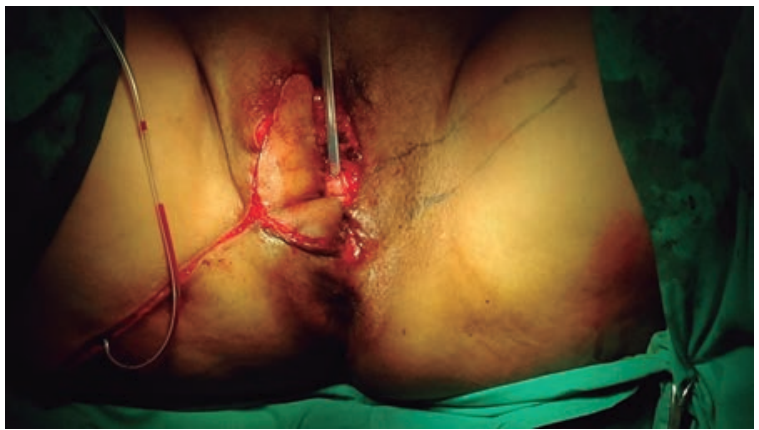

Figura 9.

Por otra parte del lado izquierdo se confeccionó un colgajo dermograso en flor de loto. Se diseco el colgajo hasta el plano aponeurótico, se fijó a mucosa vaginal en hora 12 con Vicryl. El cierre se completó de la misma manera que el colgajo contralateral. Se colocó drenaje laminar (Figura 10).

La paciente presentó buena evolución posoperatoria, al 2 do día se retiró drenaje laminar y al 3er día se retiró drenaje aspirativo. No presentó complicaciones (Figura 11).

\section{DISCUSIÓN}

El cáncer de vulva presenta una incidencia de 3-4\% de todos los cánceres ginecológicos. El 90\% corresponde al carcinoma epidermoide, seguido por melanoma y enfermedad de Paget extramamaria entre los más frecuentes ${ }^{1}$. El

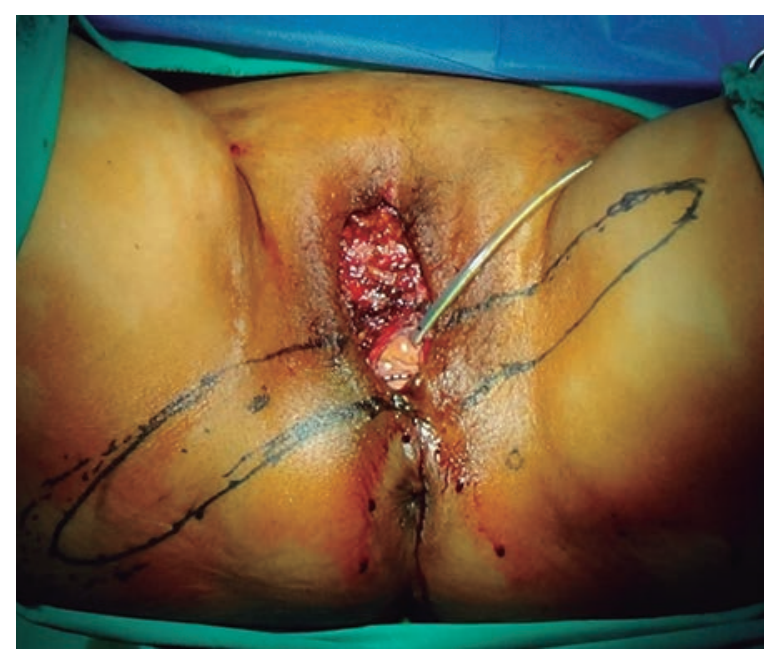

Figura 6.

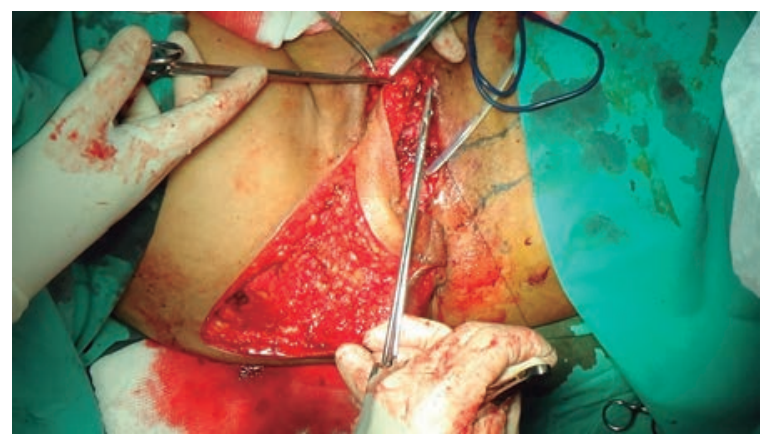

Figura 8.

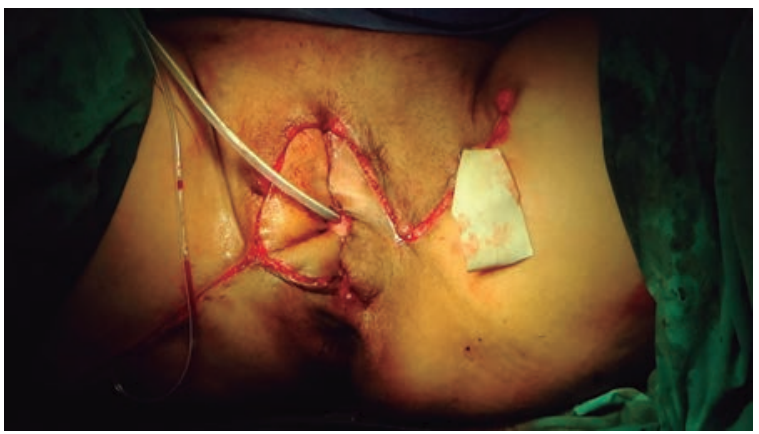

Figura 10.

tratamiento del mismo produce un gran defecto vulvar y/o vulvoperineal el cual crea un impacto biopsicosocial importante en la paciente, por lo que es un desafío para el cirujano plástico la reparación de dicha región.

Se han descripto múltiples tipos de cierre, entre ellos el cierre simple, colgajos locales, libres, injertos, terapia VAC y curación por segunda.

La región vulvoperineal tiene una gran ventaja con respecto a otras, que se debe a la gran red anastomótica tanto arterial como venosa que brindan seguridad a la hora de la reconstrucción.

El muslo ofrece, para la confección de colgajos, una vasta red de arterias perforantes dependientes de la pudenda intera y se puede preservar el nervio pudendo con algunas ramas del femorocutáneo ${ }^{2,5}$.

Saito et al. (2009) han demostrado la versatilidad del 


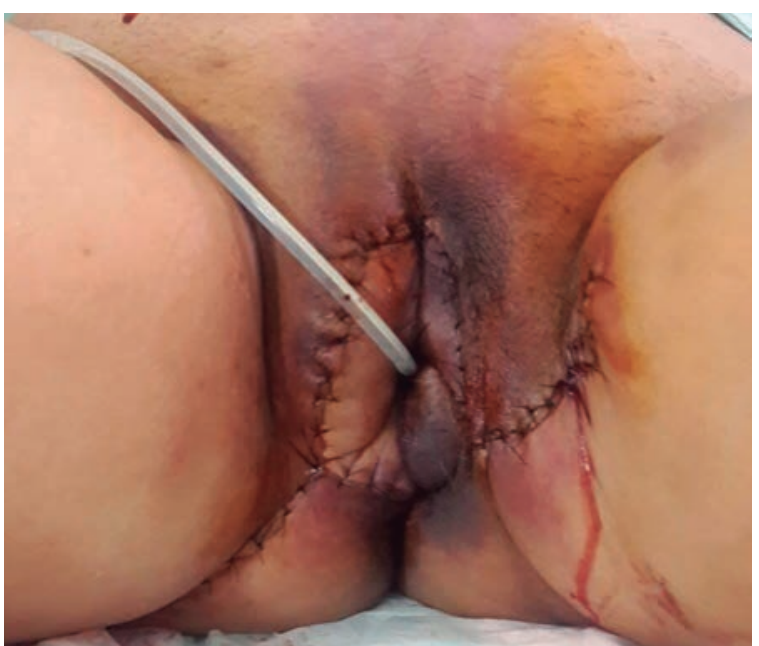

Figura 11.

colgajo de avance V-Y de muslo sin complicaciones y con aceptable resultado estético funcional ${ }^{3}$.

Winterton et al. realizaron un estudio retrospectivo descriptivo entre el año 2007 y 2010 en una población de 77 pacientes con colgajo glúteo, donde demostraron diferencias significativas en la cicatrización ante la presencia de comorbilidades y la realización de radioterapia, con bajo índice de complicaciones, entre ellas infección del sitio quirúrgico y dehiscencia. ${ }^{4}$

Coltro et al. en el año 2015 estudiaron 25 pacientes en forma retrospectiva donde realizaron colgajos de transposición dependientes de la arteria pudenda interna con conservación del nervio pudendo en el colgajo. De esta manera no solo lograron un buen resultado estético sino que también funcional al mantener la inervación de la región ${ }^{2}$.

Coiffman propone el colgajo fasciocutáneo pudendo femoral o colgajo de Singapur cuando los defectos involucran solo la pared vaginal anterior o lateral sin la necesidad de aportar volumen. Sin embargo, para defectos de la pared posterior donde se requiere aportar volumen la opción es el colgajo miocutáneo de recto abdominal pediculado. Y para los defectos que involucran la totalidad de la vagina utiliza colgajo de Málaga o de gracilis5.
Panici et al. estudiaron prospectivamente 29 pacientes que fueron intervenidas quirúrgicamente entre 2008 y 2012 por cáncer vulvar resecable. Se les realizó un colgajo glúteo de avance en V-Y y se las comparó con un grupo control al cual se le realizó cierre directo. Obtuvieron como resultado que la reconstrucción vulvar con colgajo de avance en V-Y es un procedimiento seguro, que reduce no solo la estadía hospitalaria sino también el porcentaje de complicaciones como necrosis, dehiscencia e incontinencia urinaria ${ }^{6}$.

Entre 2008 y 20011 Bondi et al. realizaron la técnica lotus petal flap en 5 pacientes con cáncer vulvar. Con los mismos, seguidos de forma prospectiva, demostraron los buenos resultados estéticos de la técnica, la ausencia de complicaciones como necrosis o infecciones urinarias y la posibilidad de una técnica libre de tensión con márgenes suficientes para evitar recidivas locales?.

Bistoletti et al. evaluaron 7 pacientes entre los años 2000 y 2002. Propusieron un colgajo relativamente fino, con buena vascularización, conservación de la sensibilidad y buen resultado estético. Los colgajos realizados eran dependientes de la arteria pudenda interna y externa. Agregaron al diseño una Z-plastia para mejorar el cierre y la transposición de los colgajos. Solo ocurrió una complicación, la cual fue dehiscencia de la sutura por infección del sitio quirúrgico. Concluyeron que el diseño del colgajo modificado con la Z-plastia mejora el arco de transposición y cierre del colgajo incluyendo el pedículo neurovascular. Como consecuencia de esto, el colgajo presenta gran versatilidad y una conservación tanto de la sensibilidad como del aporte vascular ${ }^{8}$.

\section{CONCLUSIÓN}

La confección de colgajos locales en la región vulvar para la reconstrucción de defectos es una opción a tener en cuenta debido al aporte vascular, la presencia de perforantes dependientes de la arteria pudenda, la conservación nerviosa, el bajo índice de morbilidad y el aceptable resultado estético-funcional.

\section{BIBLIOGRAFÍA}

1. International Federation of Gynecologyand Obstetrics Annual report of the results of treatment in gynecological cancer. FIGO World Congress of Gynecology and Obstetrics in Rome, Italy, in October 2012.

2. Coltro P, Ferreira M et Al. Evaluation of cutaneous sensibility of the internal pudendal artery perforator (IPAP) flap after perineal reconstruction. Journal of Plastic, Reconstructive \& Aesthetic Surgery.68, $252-261.2015$.

3. Saito A, Sawaizumi M, et Al. Stepplader $V$-Y advancement medial thigh flap for the reconstruction of vulvoperineal region. Journal of Plastic, Reconstructive \& Aesthetic Surgery. 62, 196 -199. 2009.

4. Winterton $R$, Lambe G. Gluteal fold flaps for perineal reconstruction. Journal of Plastic, Reconstructive \& Aesthetic Surgery. 66, 397405. 2013.
5. Coiffman F. Cirugía plástica, reconstructiva y estética: Tomo 6. Cap. 595, 5394-5399.3 Ed. Ed Amolca. 2007.

6. Panici P, Di Donato V. Modified gluteal fold advancement V-Y flap for vulvar reconstruction after surgery for vulvar malignancies. Gynecologic Oncology 132, 125-129. 2014.

7. Bodin F, Weitbruch D. Vulvar reconstruction by a "supra-fascial" lotus petal flap after surgery for malignancies. Gynecologic Oncology 125, 610-613. 2012.

8. Bistoletti P, Cravino T, Zimman O, Vulvar Reconstruction With Modified Fasciocutaneous Pudendal-Thigh Flaps. Plastic Surgery Fórum, 25: 384, 2002. 